\title{
DESIGN STUDIES OF POSITRON COLLECTION FOR THE NLC*
}

\author{
Yuri K. Batygin, Vinod K. Bharadwaj, David C. Schultz, John C. Sheppard \\ Stanford Linear Accelerator Center, Stanford University, Stanford, CA 94309, USA
}

\begin{abstract}
The positron source for the NLC project utilizes a 6.2 GeV electron beam interacting in a high- $\mathrm{Z}$ positron production target. The electromagnetic shower in the target results in large energy deposition which can cause damage to the target. Optimization of the collection system is required to insure long-term operation of the target with needed high positron yield into the 6dimensional acceptance of the subsequent pre-damping ring. Positron tracking through the accelerating system indicates a dilution of the initial positron phase space density. Results of the studies on the development of the collector systems design are presented.
\end{abstract}

\section{INTRODUCTION}

The positron injector includes a production target followed by a short solenoid with a strong magnetic field (flux concentrator), a $250 \mathrm{MeV}$ linac with 0.5 Tesla focusing solenoids, a $1.73 \mathrm{GeV}$ linac with quadrupole focusing, and an energy compressor system before injection into the positron pre-damping ring (PPDR). The preliminary design of injector is presented in Ref. [1], [2], [3]. The new set of parameters is listed in Tables 1, 2. The main changes in the requirements to the injector are imposed by a new value of PPDR normalized transverse acceptance of $0.045 \pi \mathrm{m}$ rad instead of the previous value of $0.09 \pi \mathrm{m}$ rad. The ultimate goal of the collector system is to provide the highest number of positrons within the 6dimensional acceptance of the pre-damping ring.

\section{POSITRON YIELD}

As positron capture is restricted by the acceptance of the pre-dumping ring, it is convenient to select an energyinvariant 6-dimensional phase space volume and observe the dilution of positron phase space density inside this volume. The 6-dimensional volume is defined in canonical conjugate variables $\left(x, P_{x}\right),\left(y, P_{y}\right),\left(z-z_{s}, p-p_{s}\right)$, where $\mathrm{P}_{\mathrm{x}}$ and $\mathrm{P}_{\mathrm{y}}$ are the canonical momentum

$$
P_{x}=p_{x}-\frac{e B_{Z}}{2} y, \quad P_{y}=p_{y}+\frac{e B_{Z}}{2} x
$$

$\mathrm{p}_{\mathrm{x}}, \mathrm{p}_{\mathrm{y}}$ are the mechanical momentum, $\mathrm{B}_{\mathrm{z}}$ is a longitudinal magnetic field, and $z_{s}, p_{s}$ define the dynamics of synchronous particles. To insure proper capture of positrons into PPDR, the edge normalized emittance of the positron beam is selected to be $\varepsilon_{\mathrm{X}}$, $\varepsilon_{\mathrm{y}} \leq 0.03 \pi \mathrm{m} \mathrm{rad}$, bunch length $\sigma_{\mathrm{z}}=6.97 \mathrm{~mm}$ and energy

\footnotetext{
*Work supported by the Department of Energy contract DEAC03-76SF00515
}

Table 1. Parameters of the electron beam drive for the positron target.

\begin{tabular}{|l|l|}
\hline \multicolumn{1}{|c|}{ Parameter } & Value \\
\hline Energy & $6.2 \mathrm{GeV}$ \\
\hline Bunch spacing & $1.4 / 2.8 \mathrm{~ns}$ \\
\hline Bunch energy variation & $1 \% \mathrm{FW}$ \\
\hline Single bunch energy spread & $1 \% \mathrm{FW}$ \\
\hline Normalized emittance & $10^{-4} \pi \mathrm{m} \mathrm{rad}$ \\
\hline Transverse size, $\sigma_{\mathrm{x}}$ & $1.6 \mathrm{~mm}$ \\
\hline Bunch length, $\sigma_{\mathrm{z}}$ & $5 \mathrm{~mm}$ \\
\hline Particles/Bunch & $1.2 / 2.4 .10^{10}$ \\
\hline Train population uniformity & $1 \% \mathrm{FW}$ \\
\hline Bunch-to-Bunch Pop. Unif. & $2 \% \mathrm{rms}$ \\
\hline Number of Bunches & $190 / 95$ \\
\hline Repetition Rate & $120 \mathrm{~Hz}$ \\
\hline Beam Power & $271 \mathrm{~kW}$ \\
\hline
\end{tabular}

Table 2. Positron beam parameters.

\begin{tabular}{|l|l|}
\hline \multicolumn{1}{|c|}{ Parameter } & Value \\
\hline Energy & $1.98 \mathrm{GeV}$ \\
\hline Bunch spacing & $1.4 / 2.8 \mathrm{~ns}$ \\
\hline Bunch energy variation & $1 \% \mathrm{FW}$ \\
\hline Single bunch energy spread & $2 \% \mathrm{FW}$ \\
\hline Normalized emittance & $0.03 \pi \mathrm{m} \mathrm{rad}$ \\
\hline Bunch length, $\sigma_{\mathrm{z}}$ & $10 \mathrm{~mm}$ \\
\hline Particles/Bunch & $0.9 / 1.8 \cdot 10^{10}$ \\
\hline Train population uniformity & $1 \% \mathrm{FW}$ \\
\hline Bunch-to-Bunch Pop. Unif. & $2 \% \mathrm{rms}$ \\
\hline Number of Bunches & $190 / 95$ \\
\hline Repetition Rate & $120 \mathrm{~Hz}$ \\
\hline Beam Power & $58 \mathrm{~kW}$ \\
\hline
\end{tabular}

spread $\Delta \mathrm{E} / \mathrm{E}=2 \%$ at the PPDR injection energy of $\mathrm{E}=$ $1.98 \mathrm{GeV}$. The value of energy spread corresponds to momentum spread of the beam $\Delta(\beta \gamma)=80$. The positron yield is defined as a ratio of positrons in a volume of phase space per incident electron at the target,

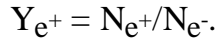

\section{BEAM DYNAMICS IN INJECTOR}

The initial positron distribution was obtained via Monte-Carlo simulation of electron-positron pair production utilizing the code EGS [4]. The positron beam created by an electromagnetic shower in solid target possesses large momentum spread. The typical value of transverse positron emittance after the interaction of the incident $6.2 \mathrm{GeV}$ electron beam of $\sigma_{\mathrm{x}, \mathrm{y}}=1.6 \mathrm{~mm}$ with a 
W-Re target of 4 radiation lengths (RL) is $\varepsilon_{\mathrm{O}}=0.135 \pi \mathrm{m}$ rad. The production target is in the field of a tapered solenoid with a maximum field of $\mathrm{B}_{\mathrm{t}}=1.2$ Tesla. The presence of a magnetic field at the target does not significantly increase the normalized beam emittance:

$$
\varepsilon=\sqrt{\varepsilon_{\mathrm{o}}^{2}+\left(\frac{\mathrm{e} \mathrm{B}_{\mathrm{t}} \mathrm{R}_{\mathrm{t}}^{2}}{2 \mathrm{mc}}\right)^{2}} \approx \varepsilon_{\mathrm{o}} .
$$

The created beam enters the strong longitudinal magnetic field of a flux concentrator. The flux concentrator is a solenoid with sharp increase of magnetic field up to peak value of 5.8 Tesla at a distance of $5 \mathrm{~mm}$ from the target and an adiabatic decrease of the field over a distance of $15 \mathrm{~cm}$ [5]. The strong magnetic field at the target is required to confine the emitted positron beam with its large momentum spread.

The flux concentrator is followed by a $250 \mathrm{MeV}$ linac with an effective accelerating gradient of $25 \mathrm{MeV} / \mathrm{m}$ and transverse focusing by a 0.5 Tesla solenoid field. The transverse acceptance of the linac with solenoid focusing is energy independent:

$$
\alpha=\frac{\mathrm{eB}_{\mathrm{Z}}}{2 \mathrm{mc}} \mathrm{a}^{2}=0.06 \pi \mathrm{m} \mathrm{rad} .
$$

After acceleration to $250 \mathrm{MeV}$ positrons are accelerated in a linac with quadrupole focusing and an accelerating gradient of $15 \mathrm{MeV} / \mathrm{m}$.

Tables 3 and Fig. 1 show the results of the positron beam dynamics simulation in the injector. The positron yield within the $6 \mathrm{D}$ phase space volume drops from the value of 4.7 at the target to 0.76 at the end of the linac. One of the reasons for the dilution of the beam phase space density in the linac is the beam energy spread, which results in chromaticity-induced beam emittance growth. Calculations were done for two cases, using longitudinal focusing: for a linac with solenoidal focusing with $\mathrm{B}=0.5 \mathrm{Tesla}$, and a linac with focusing by periodic permanent magnets (PPM). The field of the PPM was approximated by function

$$
\mathrm{B}_{\mathrm{Z}}=\mathrm{B}_{\mathrm{o}} \sin \left(\frac{2 \pi \mathrm{z}}{\mathrm{L}}\right),
$$

where $\mathrm{B}_{\mathrm{o}}=0.5$ Tesla is the maximum field and $\mathrm{L}=50 \mathrm{~cm}$ was chosen to be the period of the PPM structure. Particle tracking indicates that in the case of longitudinal focusing, the positron yield at the end of the linac is increased to $\mathrm{Y}_{\mathrm{e}^{+}}=1.15$.
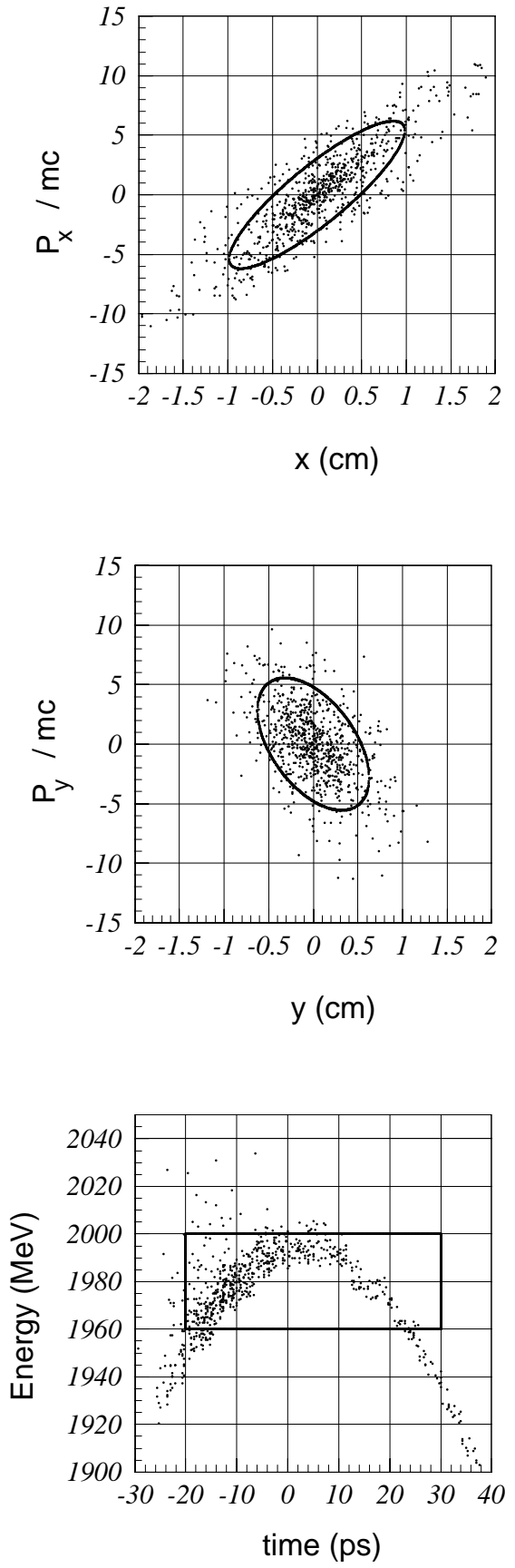

Fig. 1. Positron distribution at $1.98 \mathrm{GeV}$.

Table 3. Positron yield along the injector.

\begin{tabular}{|l|c|c|c|c|}
\hline & After target & $\begin{array}{c}\text { After flux } \\
\text { concentrator }\end{array}$ & At 250 MeV & At 1.9 GeV \\
\hline Positron yield & 12 & 4.7 & 2.2 & 1.9 \\
\hline $\begin{array}{l}\text { Fraction of } \\
\text { positrons within 6- } \\
\text { D phase space }\end{array}$ & 0.39 & 0.37 & 0.74 & 0.40 \\
\hline $\begin{array}{l}\text { Positron yield } \\
\text { within 6-D phase } \\
\text { space }\end{array}$ & 4.70 & 1.76 & 1.60 & 0.76 \\
\hline
\end{tabular}




\section{DYNAMICS OF POLARIZED POSITRONS}

One option for the positron injector being considered is the collection and acceleration of polarized positrons. Polarized positrons may be produced by targeting helically polarized gammas, which could be made by Compton backscattering [6] or with a helical undulator [7], on a thin target. To study the effects of positron depolarization, the particle tracking code was modified to include Thomas-BMT equation [8], describing the precession of the spin vector $\overrightarrow{\mathrm{S}}$ :

$$
\frac{\overrightarrow{\mathrm{dS}}}{\mathrm{dt}}=\frac{\mathrm{e} \overrightarrow{\mathrm{S}}}{\mathrm{m} \gamma} \times\left[(1+\mathrm{G} \gamma) \overrightarrow{\mathrm{B}}_{\perp}+(1+\mathrm{G}) \overrightarrow{\mathrm{B}}_{\mathrm{II}}+\left(\mathrm{G} \gamma+\frac{\gamma}{1+\gamma}\right) \frac{\overrightarrow{\mathrm{Ex}}}{\mathrm{c}}\right],(
$$

where $G$ is the anomalous magnetic moment of the positron, $\vec{E}$ is the electrical field, and $\vec{B}_{\perp}$ and $\vec{B}_{I I}$ are components of the magnetic field perpendicular and parallel to the particle velocity. The spin advance at a small distance $\delta z$ is described as a matrix [9] :

$$
\left|\begin{array}{c}
S_{\mathrm{X}} \\
\mathrm{S}_{\mathrm{y}} \\
\mathrm{S}_{\mathrm{Z}}
\end{array}\right|=\left|\begin{array}{ccc}
1-\mathrm{a}\left(\mathrm{B}^{2}+\mathrm{C}^{2}\right) & \mathrm{ABa}+\mathrm{Cb} & \mathrm{ACa}-\mathrm{Bb} \\
\mathrm{ABa}-\mathrm{Cb} & 1-\mathrm{a}\left(\mathrm{A}^{2}+\mathrm{C}^{2}\right) & \mathrm{BCa}+\mathrm{Ab} \\
\mathrm{ACa}+\mathrm{Bb} & \mathrm{BCa}-\mathrm{Ab} & 1-\mathrm{a}\left(\mathrm{A}^{2}+\mathrm{B}^{2}\right)
\end{array}\right|\left|\begin{array}{c}
\mathrm{S}_{\mathrm{X}, \mathrm{o}} \\
\mathrm{S}_{\mathrm{y}, \mathrm{o}} \\
\mathrm{S}_{\mathrm{z}, \mathrm{o}}
\end{array}\right|,
$$

$$
\begin{gathered}
A=\frac{D_{x}}{D_{0}}, \quad B=\frac{D_{y}}{D_{o}}, \quad C=\frac{D_{z}}{D_{o}}, \quad D_{0}=\sqrt{D_{x}^{2}+D_{y}^{2}+D_{z}^{2}}, \\
a=1-\cos \left(D_{o} \delta z\right), \quad b=\sin \left(D_{o} \delta z\right),
\end{gathered}
$$

where components $\mathrm{D}_{\mathrm{x}}, \mathrm{D}_{\mathrm{y}}, \mathrm{D}_{\mathrm{z}}$ are defined by the equations:

$$
\begin{gathered}
D_{x}=\frac{e}{m \gamma v}\left[(1+G \gamma)\left(B_{x}-x^{\prime} B_{z}\right)+(1+G) x^{\prime} B_{z}+\right. \\
\left.\frac{v}{c^{2}}\left(\frac{\gamma}{1+\gamma}+G \gamma\right)\left(E_{y}-y^{\prime} E_{z}\right)\right] \\
D_{y}=\frac{e}{m \gamma v}\left[(1+G \gamma)\left(B_{y}-y^{\prime} B_{z}\right)+(1+G) y^{\prime} B_{z}+\right. \\
\left.\frac{v}{c^{2}}\left(\frac{\gamma}{1+\gamma}+G \gamma\right)\left(x^{\prime} E_{z}-E_{x}\right)\right] \\
D_{z}=\frac{e}{m \gamma v}\left[(1+G \gamma)\left(-x^{\prime} B_{x}-y^{\prime} B_{y}\right)+(1+G) .\right. \\
\left.\left(x^{\prime} B_{x}+B_{z}+y^{\prime} B_{y}\right)+\frac{v}{c^{2}}\left(\frac{\gamma}{1+\gamma}+G \gamma\right)\left(y^{\prime} E_{x}-E_{y} x^{\prime}\right)\right]
\end{gathered} .
$$

Fig. 2 illustrates the polarization of positrons emerging from 0.2 RL W-Re target as a function of their energy, calculated by the EGS code extended to study polarization propagation in the target [10]. Polarization is defined as the probability to find the spin of the positron along the direction of the positron momentum.

During beam transport and acceleration, the spin vector precesses, resulting in the depolarization of the beam. We define the longitudinal polarization as an average of the product of the longitudinal component $\mathrm{S}_{\mathrm{Z}}$

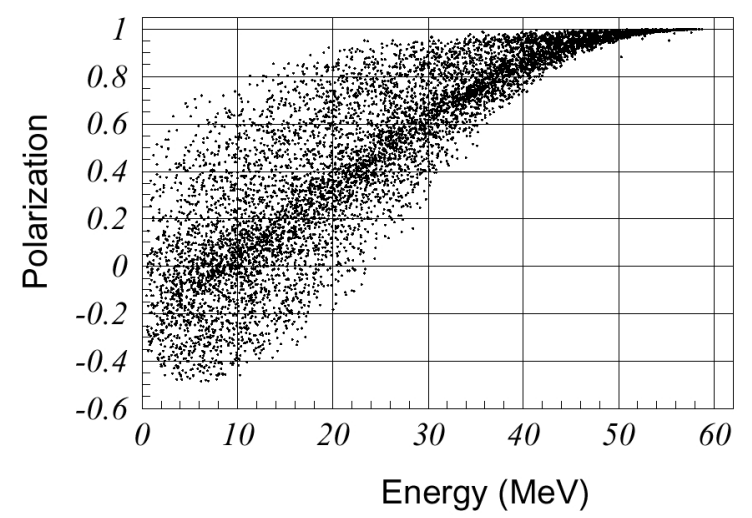

Fig. 2. Initial positron polarization from $60 \mathrm{MeV} \gamma$ rays.

Table 4. Polarization of positrons at $250 \mathrm{MeV}$ captured within the 6-D phase space.

\begin{tabular}{|c|c|c|}
\hline $\begin{array}{c}\text { Energy range, } \\
\mathrm{MeV}\end{array}$ & $\begin{array}{c}\text { Positron capture } \\
\text { within 6D phase } \\
\text { space }\end{array}$ & $\left\langle\mathrm{P}_{\mathrm{Z}}\right\rangle$ \\
\hline $260-280$ & 0.028 & 0.92 \\
\hline $255-280$ & 0.038 & 0.86 \\
\hline $245-280$ & 0.084 & 0.68 \\
\hline $242-280$ & 0.11 & 0.60 \\
\hline $240-280$ & 0.14 & 0.53 \\
\hline
\end{tabular}

and the value of polarization, $\mathrm{P}$, over all positrons:

$$
\left\langle\mathrm{P}_{\mathrm{Z}}\right\rangle=\frac{1}{\mathrm{~N}} \underset{\mathrm{i}=1}{\mathrm{~N}} \mathrm{~S}_{\mathrm{Z}}^{(\mathrm{i})} \mathrm{P}^{(\mathrm{i})}
$$

Table 4 illustrates the distribution of polarized positrons at $250 \mathrm{MeV}$. Simulation shows that $11 \%$ of the produced positrons have an average value of polarization of $60 \%$. Additional study is required to increase the number of captured positrons keeping the value of polarization high, and determine the needed $\gamma$ flux.

\section{REFERENCES}

[1] Zeroth-Order Design Report for the Next Linear Collider, SLAC-Report 474, (1996).

[2] T.Kotseroglou et. al., Proceedings of the 1999 Particle Accelerator Conference, Editors: A.Luccio, W.MacKay, 3450 (1999).

[3] J.C. Sheppard et. al., "Update to the NLC Injector System Design", PAC 2001, June 2001.

[4] W.Nelson, H.Hirayama and D.Rogers, "The EGS4 Code System", SLAC-Report-265 (1985).

[5] A.V.Kulikov, S.D.Ecklund and E.M.Reuter, Proceeding of the 1991 Particle Accelerator Conference, 2005 (1991).

[6] T.Omori, Proc. of the Workshop on New Kind of Positron Sources, Stanford, (1997), SLAC-R-502, p. 285.

[7] A.Mikhailichenko, Proc. of the Workshop on New Kind of Positron Sources, Stanford, (1997), SLAC-R-502, p.229.

[8]V.Bargmann, L.Michel, V.L.Telegdi, Phys. Rev. Lett. 2 (1959) 435 .

[9] Y.Batygin and T.Katayama, Physical Review E, Vol. 58, (1998), 1019.

[10] K.Flottmann, Ph.D. Thesis, DESY-93-161A (1993). 